\title{
Optical Properties of Metal Nanoshells
}

\author{
Encai Hao, ${ }^{\dagger}$ Shuyou Li, $\stackrel{\dagger}{ }$ Ryan C. Bailey, ${ }^{\dagger}$ Shengli Zou,${ }^{\dagger}$ George C. Schatz,,${ }^{*} \dagger$ and \\ Joseph T. Hupp*,† \\ Department of Chemistry and Institute for Nanotechnology and Department of Materials Science and \\ Engineering, Northwestern University, 2145 Sheridan Road, Evanston, Illinois 60208
}

Received: August 5, 2003; In Final Form: October 29, 2003

\begin{abstract}
We present a combination of theory and experiment designed to elucidate the properties of gold nanoshells. Wet chemistry methods are used to prepare the nanoshells, and transmission electron microscopy (TEM) analysis is used to characterize the shell structure, demonstrating the presence of pinholes in the shells. Both Mie theory and the discrete dipole approximation (a numerical method) are used to characterize the electrodynamics of the shell structures, including both perfect and pinhole defected shells. The calculations show that $2-5 \mathrm{~nm}$ pinholes have only a small effect on the extinction spectra; however, they lead to local electric fields that are enhanced by a factor of 3-4 close to the plasmon maximum. This makes metal nanoshells (with holes) attractive materials for surface enhanced Raman spectroscopy applications.
\end{abstract}

\section{Introduction}

There have been several important recent discoveries concerning the use of wet-chemical methods to make in high-yield dispersed gold and silver colloidal particles with well-defined structures other than solid spheres. These include nanoscale rods, ${ }^{1-4}$ triangular prisms,,${ }^{5,6}$ disks, ${ }^{7,8}$ and nanoshells. ${ }^{9-11}$ Some of these synthetic approaches have involved transforming initially prepared solid spheres into one of these shapes, and when this happens, the surface plasmon resonances are strongly affected, typically red-shifting and even splitting into distinctive dipole and quadrupole plasmon modes. ${ }^{3,5,12}$ This has caused extensive interest in using these particles in photonic materials and biosensors.

To characterize these intriguing optical features, a number of theoretical approaches have been developed, including the discrete dipole approximation (DDA),${ }^{12-15}$ finite difference time domain methods (FDTD), ${ }^{16}$ the multiple multipole method, ${ }^{17}$ and the modified long wavelength approximation (MLWA). ${ }^{12,13}$ Among them, the DDA is a particularly useful technique for describing isolated nanoparticles with arbitrary shape and a complex surrounding environment (solvent, substrate, other nearby particles). ${ }^{12}$ In the DDA, the object of interest is represented as a cubic array of $N$ polarizable elements. The response of this array to an applied electromagnetic field is then described by self-consistently determining the induced dipole moment in each element. ${ }^{13}$ This information can be used to determine far-field properties such as extinction efficiencies and also near-field properties especially the electromagnetic field (E-field) near the particle surface. ${ }^{12,14,15}$

Metallic nanoshells are one of the most interesting and possibly useful of the recently developed nanoparticle structures. $^{9-11,18-20}$ These particles usually consist of a thin metallic (Au or Ag) shell around a dielectric core (such as $\mathrm{SiO}_{2}$ or polystyrene). ${ }^{9,11}$ Very recently, Halas and co-workers ${ }^{21}$ have developed a new strategy to produce reduced-symmetry metallodielectric nanoparticles such as nanocups and nanocaps. Sun and $\mathrm{Xia}^{22}$ reported a simple approach to the fabrication of gold

* To whom correspondence should be addressed. E-mail: schatz@ chem.northwestern.edu (G.C.S.); jthupp@ chem.northwestern.edu (J.T.H.).

Department of Chemistry and Institute for Nanotechnology.

$\doteqdot$ Department of Materials Science and Engineering. nanoshells having hollow interiors by reacting aqueous $\mathrm{HAuCl}_{4}$ solutions with silver nanoparticles. The resulting nanoshells have plasmon resonances that are strongly red-shifted compared to solid gold spheres, with a wavelength that is tunable depending on the ratio of the shell thickness to its diameter. ${ }^{20}$ This tunable optical feature makes nanoshells attractive for applications to extinction $^{22}$ or SERS-based sensing. ${ }^{11,19}$

In this paper, we prepare gold nanoshell particles with hollow interiors using an approach similar to the published method. ${ }^{22}$ Interestingly, we find that pinholes (few nanometers in size) are present on the gold nanoshells. These pinholes could then serve as cavities in which trapped molecules might have possibly interesting, but unknown, consequences for extinction and SERS. Although the extinction spectrum of a perfect nanoshell can easily be determined by Mie theory, the influence of pinholes in the shell surface cannot. To address this problem, we use the DDA method to study pinhole-defected nanoshells, determining both far-field (extinction) and near-field (local field) effects. We find that while the pinhole does not affect the extinction spectra of gold nanoshells, it does create local E-field enhancements that can serve as hot sites for SERS.

\section{Experimental Section}

$\mathrm{Au}$ nanoshells have been prepared by reacting $\mathrm{HAuCl}_{4}$ with silver nanoparticles similar to the method published by Sun and $\mathrm{Xia}^{22}$ Briefly, Ag nanoparticles were prepared by injection of $\mathrm{NaBH}_{4}(50 \mathrm{mM}, 2 \mathrm{~mL})$ to an aqueous solution of $\mathrm{AgNO}_{3}(0.2$ $\mathrm{mM}, 100 \mathrm{~mL})$ in the presence of sodium citrate $(0.5 \mathrm{mM})$. The resulting Ag colloid was then kept at $70{ }^{\circ} \mathrm{C}$ for $2 \mathrm{~h}^{23}$ While stirring, $0.68 \mathrm{~mL}$ of $\mathrm{HAuCl}_{4}(0.1 \mathrm{M})$ was added to $100 \mathrm{~mL}$ of the Ag colloid solution. A color change from yellow to red to dark blue was observed during the course of the replacement reaction. After $1 \mathrm{~h}$ of stirring, the particles were purified by gradient centrifugation, washed twice using an aqueous solution of sodium citrate $(0.3 \mathrm{mM})$, redispersed in $5 \mathrm{~mL}$ of sodium citrate $(0.3 \mathrm{mM})$, and finally kept at $4{ }^{\circ} \mathrm{C}$.

The resulting particles were characterized optically using a Cary 5 spectrophotometer and structurally with a Hitachi HF 2000 TEM and a Molecular Imaging PicoSPM operating in contact mode. 


\section{Discrete Dipole Approximation (DDA) Calculations}

The DDA method has been described in detail elsewhere. ${ }^{12-15}$ The induced dipole polarization $P_{i}$ in each cubic element is determined from

$$
P_{i}=\alpha_{i} \mathbf{E}_{\mathrm{loc}}\left(r_{i}\right)
$$

where $\alpha_{i}$ and $r_{i}$ are the polarizability and location of the $i$ th dipole, and $\mathbf{E}_{\text {loc }}$ is the local electric field. The local field at each dipole is given by

$$
\mathbf{E}_{\mathrm{loc}, i}=E_{0} \exp \left(i k r_{i}\right)-\sum_{j \neq i} A_{i j} P_{j}
$$

where $E_{0}$ and $k$ are the amplitude and wave vector of the incident field, respectively. The interaction matrix $A$ has the form

$$
\begin{aligned}
& A_{i j} \cdot P_{j}= \\
& \frac{\exp \left(i k r_{i j}\right)}{r_{i j}{ }^{3}}\left\{k^{2} r_{i j}\left(r_{i j} P_{j}\right)+\frac{\left(1-i k r_{i j}\right)}{r_{i j}{ }^{2}}\left[r_{i j}{ }^{2} P_{j}-3 r_{i j}\left(r_{i j} P_{j}\right)\right]\right\}_{(j \neq i)}
\end{aligned}
$$

where $k=\omega / c$. Substituting eqs 2 and 3 into 1 , we can we generate the system of equations

$$
\mathrm{A}^{\prime} \cdot \mathrm{P}=\mathrm{E}
$$

where the off diagonal elements of the matrix, $A_{i j}{ }^{\prime}$, are same as $A_{i j}$, and the diagonal elements of the matrix, $A_{i i}{ }^{\prime}$, are $\alpha^{-1}$.

For a system with $N$ total dipoles, $E$ and $P$ are $3 N$-dimensional vectors, and $A^{\prime}$ is a $3 N \times 3 N$ matrix. Solving this set of $3 N$ complex linear equations, the polarizations $P$ are determined, and from this we derive the extinction cross-section $\left(C_{\text {ext }}\right)$ using

$$
C_{\mathrm{ext}}=\frac{4 \pi k}{\left|E_{0}\right|^{2}} \sum_{i=1}^{N} \operatorname{Im}\left(E_{\mathrm{inc}, i} * \cdot P_{j}\right)
$$

All calculations here refer to water as the external dielectric medium for both inside and outside of $\mathrm{Au}$ nanoshells. The dielectric constant in all calculations has been taken from Palik ${ }^{24}$ but smoothed as described by Jensen et al. ${ }^{15}$ All of the calculations refer to a DDA grid size of $1 \mathrm{~nm}$. In previous work, ${ }^{25}$ we demonstrated that the E-fields obtained using the DDA approach are converged to within a factor of 3 for grid sizes of $1 \mathrm{~nm}$ compared to what is obtained from Mie theory. More accurate results can be obtained using finer grids (essentially quantitative results arise for a grid size of $0.25 \mathrm{~nm}$ ) however the substantial additional expense is not crucial for the present application where the relative sizes of enhancements are of primary interest.

\section{Results and Discussion}

IV.A. Characterization. In past work, silver nanoparticles including spheres, ${ }^{22}$ rods, ${ }^{26}$ cubes, ${ }^{27}$ and triangular prisms ${ }^{28,29}$ have been etched with $\mathrm{HAuCl}_{4}$ to generate hollow particles. Sun and Xia generated seamless Au nanoshells by combining the replacement reaction with annealing. ${ }^{22}$ Initially, the addition of $\mathrm{HAuCl}_{4}$ to an aqueous solution of silver nanoparticles leads to a replacement reaction with a molar $\mathrm{Au} / \mathrm{Ag}$ ratio of $0.33: 1$. The elemental Au nucleates and evolves into a thin shell around the Ag template. As shown in Figure 1a (as well as Figure S1 in the Supporting Information), the Au nanoshells have a broad size distribution ranging from 20 to $40 \mathrm{~nm}$. The size distribution
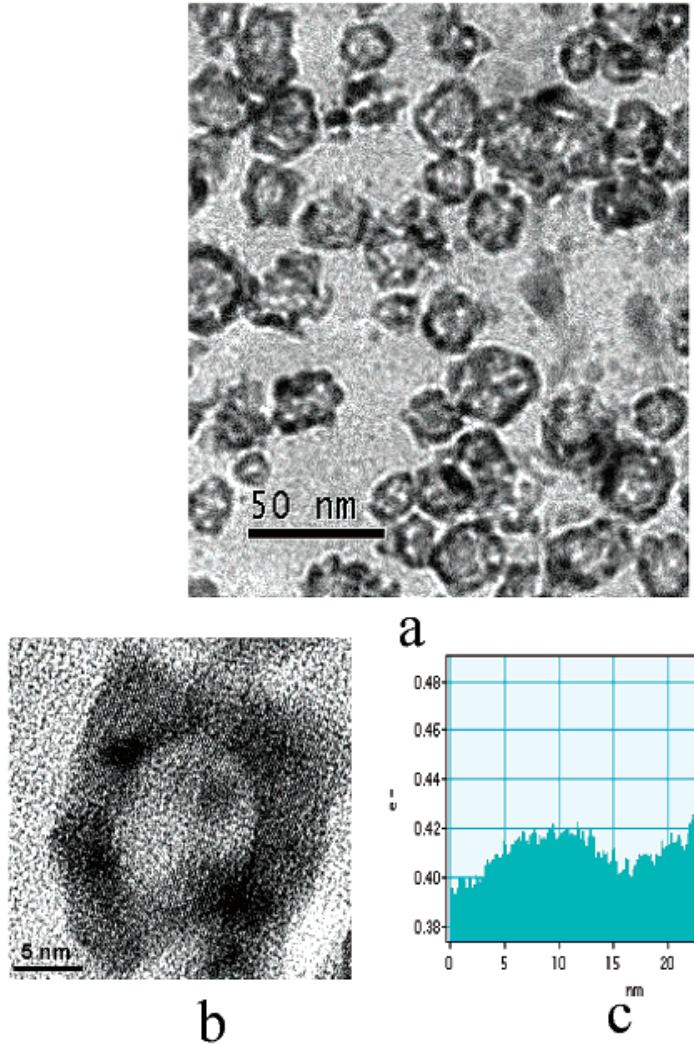

$\mathrm{a}$

Figure 1. (a) TEM photograph of Au nanoshell, (b) high-resolution TEM photograph of individual Au nanoshell, and (c) intensity linescan across a gold elemental map of particle shown in Figure 1b.

of nanoshells match that of the Ag nanoparticle templates. An important feature of these particles is the presence of pinhole (several nm) structures, which may allow both $\mathrm{HAuCl}_{4}$ and $\mathrm{Ag}^{+}$ to continuously diffuse across the shell until the entire $\mathrm{Ag}$ template has been etched. Because the TEM image is a 2-dimension view of the nanoparticles, the white spots on nanoshells represent the smallest size of holes, where the electron beams could go through from top to bottom of the nanoshells. The HRTEM image (Figure 1b) of an individual Au nanoshell shows that the shell thickness for this particle is about $3.0 \mathrm{~nm}$. Figure 1c is an intensity line-scan of elemental gold across an individual particle using the Gatan elemental mapping technique. ${ }^{30}$ This confirms the hollow structure with high gold intensity seen at the edge and lower in the center.

The nanoshells were further characterized using atomic force microscopy (AFM) using contact mode. Au nanoshells were dispersed onto a clean silicon surface and dried in air. Although tip-limited lateral resolution limits the characterization of nanoshell shape, a cross-sectional line scans yields particle diameters, assuming a spherical shape. ${ }^{31}$ AFM measurements (see Figure S2 in the Supporting Information) are consistent with TEM results verifying the particle dimensions.

Figure $2 \mathrm{a}$ presents extinction spectra for the initial silver particles and the final gold shells. The shell spectra show a broad maximum at $736 \mathrm{~nm}$ that we will analyze in the next section.

IV.B. Theoretical: Extinction Spectra. It has been demonstrated both experimentally and theoretically that nanoshells exhibit interesting extinction spectra that are strongly dependent on size and shell thickness. ${ }^{9,11,20,22}$ Sometimes these nanoshells have pinhole-like defects. ${ }^{11,32}$ However, it is not known how the optical properties of the nanoshells depend on the pinhole 

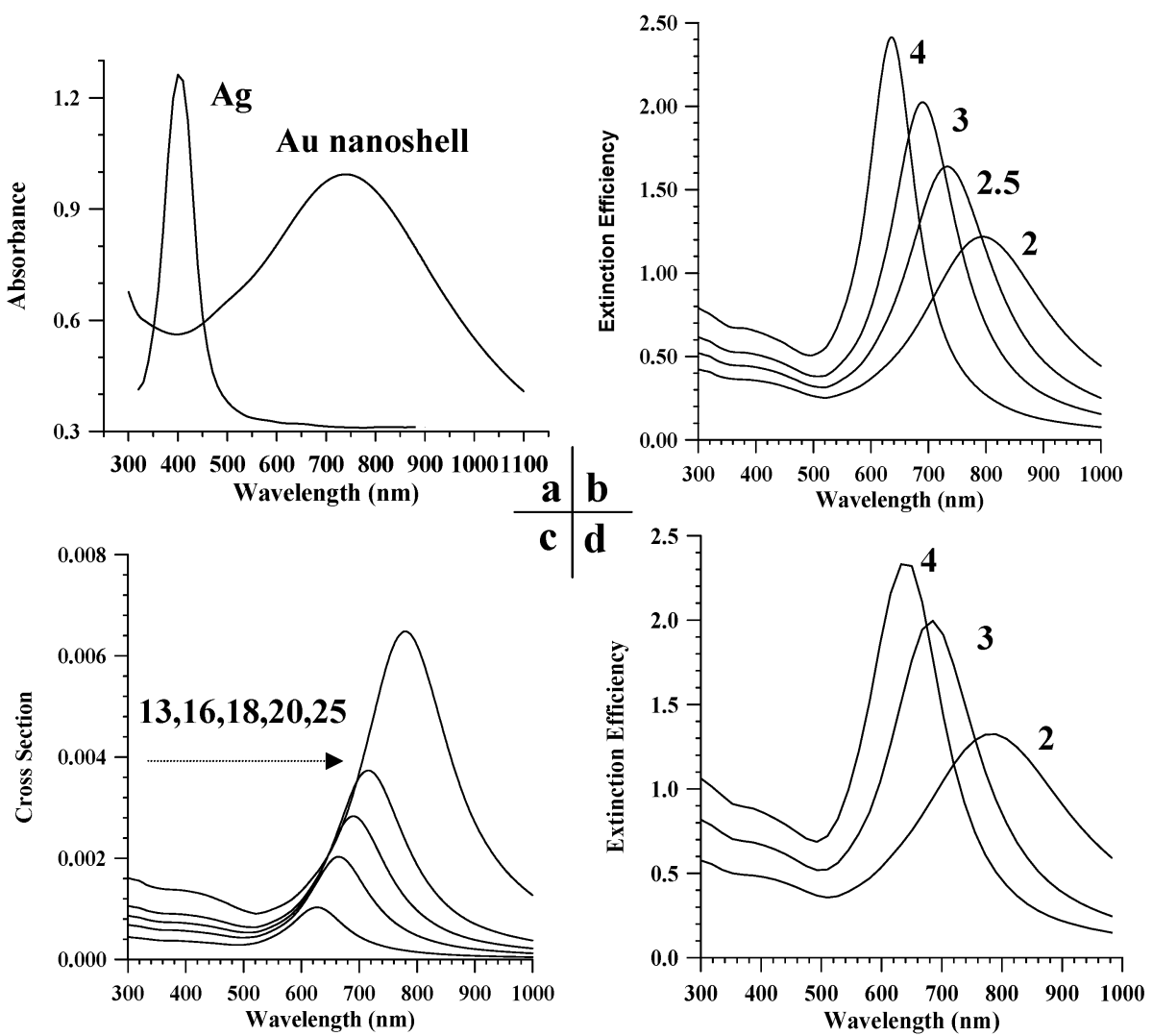

Figure 2. (a) UV-visible spectrum of a solution of Au nanoshells, (b) the Mie theory calculated extinction spectra of Au nanoshells (18 nm in radii) with varying shell thickness, (c) the Mie theory calculated extinction spectra of Au nanoshells ( $3 \mathrm{~nm}$ in thickness) as function of particle radius, (d) the DDA calculated extinction spectra of Au nanoshells (18 $\mathrm{nm}$ in radii) with varying shell thickness.

structures. To provide insight into this, we used Mie theory 33 and the DDA method ${ }^{12-15}$ to calculate the extinction spectrum of the Au nanoshells.

Figure 2, parts b and c, shows Mie theory results for perfect shells, demonstrating the well-known result that the extinction spectrum is very sensitive to the thickness and size of the nanoshells, with the plasmon resonance shifting red when the thickness is decreased and the size is increased. Note that the dielectric constant is modified for all calculations according to the shell thickness. ${ }^{34,35}$ Similar calculations performed without this correction yield extinction spectra with a sharpening and intensification features. These results agree with results for $\mathrm{Au}$ nanoshells with dielectric cores that were reported by Halas et al. ${ }^{9} 10$ and for hollow nanoshells reported by Xia et al. ${ }^{22,26}$ In addition, we see that the width of the experimental observations (Figure 2a) can be understood based on the broad distribution of nanoparticle sizes and thicknesses.

Figure $2 \mathrm{~d}$ shows that the DDA results for the perfect shells are very similar to the Mie theory results (Figure 2b), thus demonstrating that the DDA method is capable of describing the shell electrodynamics adequately. Further DDA calculations (Figure 3) that we discuss below show that pinholes $(2-5 \mathrm{~nm}$ in diameter) do not affect the extinction spectra of the nanoshells. Thus, except for inhomogeneity, the calculated perfect nanoshell spectrum for nanoshells that are $36 \mathrm{~nm}$ in diameter and $2.5 \mathrm{~nm}$ in thickness is sufficient to explain the experimental observations. This result is different from what was concluded in the nanocup studies of Charnay et al., ${ }^{21}$ who used FDTD calculations to show that cup structures $(50 \mathrm{~nm}$ hole in a $50 \mathrm{~nm}$ radius shell whose thickness is $25 \mathrm{~nm}$ ) produce plasmon resonances that are strongly shifted from the perfect

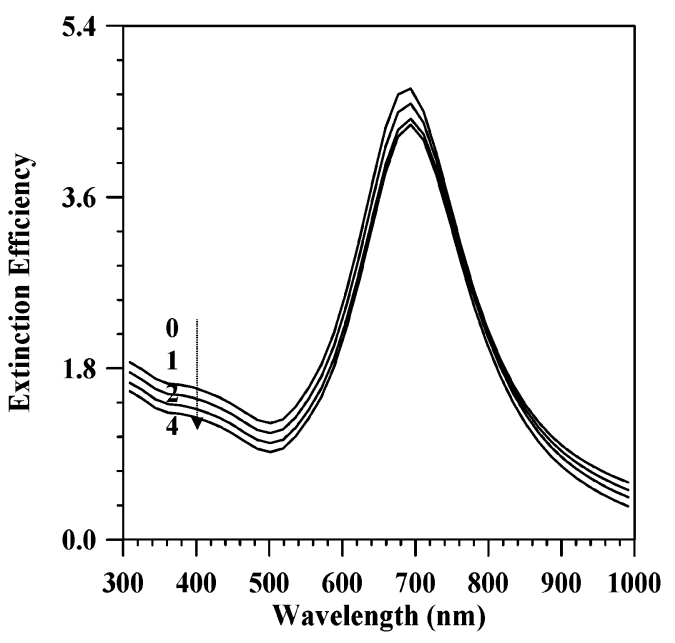

Figure 3. DDA calculated extinction spectra of Au nanoshells (18 $\mathrm{nm}$ in radii and $3 \mathrm{~nm}$ in thickness) with different numbers of holes each with $3 \mathrm{~nm}$ in diameter.

shell resonances. In this case, the influence of the hole on the shell spectrum is significant, as the area of the hole is comparable to the projected area of the shell. This contrasts with what we consider here, where our pinholes are only a few percent of the size of the shell.

IV.C. Theoretical: Local Field Effects. Metal nanoshells are considered to be attractive for applications in SERS ${ }^{19}$ and environmental sensing..$^{22}$ SERS has been the subject of extensive studies, ${ }^{19,36-38}$ and it is generally agreed that an important contribution to the SERS enhancement comes from the elec- 

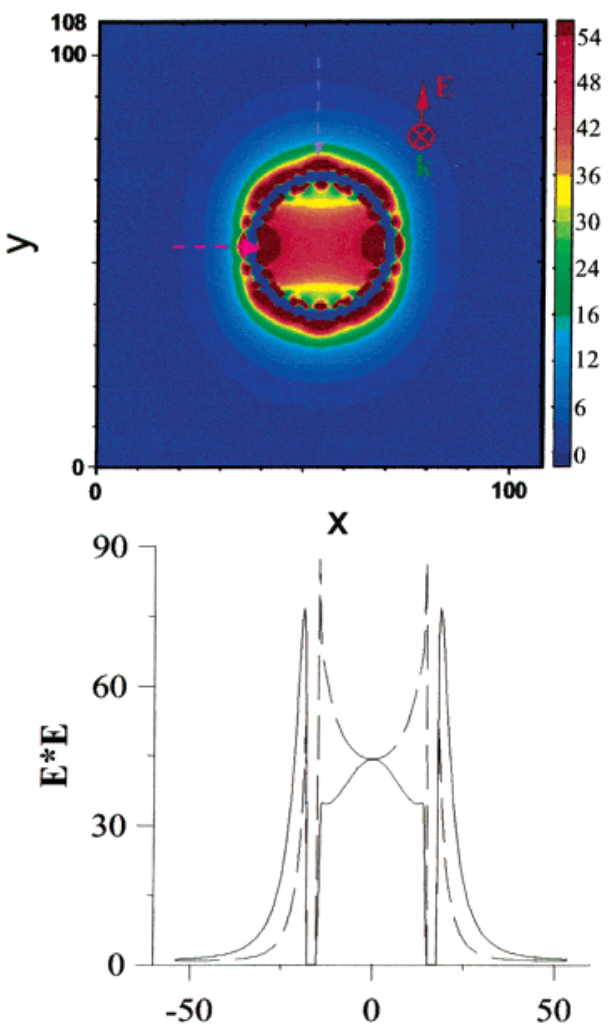

Figure 4. (a) E-field enhancement contours external to pinhole-free Au nanoshells (18 $\mathrm{nm}$ in radii and $3 \mathrm{~nm}$ in thickness, with wavelength chosen to be the plasmon maximum). (b) Line analysis of E-field enhancement for $x=0$ along the $y$ axis (solid line), and for $y=0$ along the $x$ axis (dash line). The $y$ axis represents the polarization direction. The DDA calculations use a cubic grid with a grid spacing of $1 \mathrm{~nm}$.

tromagnetic (EM) enhancement mechanism, ${ }^{39}$ in which plasmon excitation in the particle creates an enhanced E-field near the particle, which in turn leads to enhanced Raman excitation and emission. The electrodynamics of metal nanoparticles including spheroids, rods, and triangular prisms has been of interest, especially to estimates of the SERS enhancement factor. ${ }^{12,15,25}$ For perfect metal nanoshells, Mie theory has been used to determine E-field enhancements, and the correlation between particle structure and calculated SERS enhancement accurately matches measured results. ${ }^{19}$

Upon plasmon excitation, the induced polarization in a nanoshell is predominantly oriented along the applied electric polarization vector. Figure $4 \mathrm{a}$ shows electrical field (E-field) enhancement for a pinhole free nanoshell, plotted as contours of $|\mathbf{E}|^{2}$. We see large field enhancements both inside and outside of the nanoshells. For the outside E-field, the maximum enhancement occurs along the $y$ axis (i.e., along the polarization direction) and only locations within a few $\mathrm{nm}$ of the shell surface. This is a typical result for a dipole plasmon resonance. ${ }^{25}$ However, inside the shell, large fields are found for a large volume of the spherical cavity, with the maximum enhancement occurring close to the shell surface along the $x$ axis. This behavior can be understood by realizing that in the electrostatic limit (i.e., very small shells), the quasistatic (LaPlace equation) field inside the cavity is a constant (i.e., independent of position). We verified that the DDA calculations give this result for 10 $\mathrm{nm}$ diameter particles. However, for the $36 \mathrm{~nm}$ particles, finite wavelength effects lead to depolarization, and thus, the field inside the shell is mostly in the range from 20 to 50 times the applied field.
Figure $4 \mathrm{~b}$ shows a line scan of the E-field enhancement for $x=0$ along the $y$ axis (solid line), and for $y=0$ along the $x$ axis (dashed line). We see that the largest fields $\left(|\mathbf{E}|^{2}\right)$ at the outside and inside surfaces are 80 and 90 times the applied field, respectively. This is comparable to the largest fields that are found at the surfaces of spherical silver particles of comparable size. $^{12}$

Figure 5 shows the effect of the presence of one and two pinholes on the E-fields around the Au nanoshells. When the polarization is along the $y$ axis and holes are located at either $x=\mathrm{R}$ for one pinhole or $x= \pm \mathrm{R}$ for two ( $\mathrm{R}=$ the sphere radius) and with $y=z=0$, the local E-field enhancement shows a maximum at the pinhole locations. A line scan analysis of the E-field enhancement for $y=z=0$ shows a maximum around 320 times the applied field. This enhancement is about 3-4 times that of the seamless nanoshells. It turns out that even larger fields (nearly 1000 times the applied field) are obtained in Figure 5, parts b and c, for geometries slightly off the line scan. Figure 6 shows the largest E-field enhancements for the hole region associated with Figure 5b, and we see that the largest E-field is slightly displaced from the middle of the pinholes. The precise details of where the fields maximize are subtle, as for small holes the maximum field is in the middle (where the contribution of the top and bottom shell surfaces simultaneously maximize), and then it moves away from the middle to the shell surfaces as hole size increases (i.e., the contribution of one shell surface dominates over the other). This junction behavior is similar to what was previously found at the junction between two nanoparticles. ${ }^{40-43}$ When the polarization is along the $x$ axis, we find that the E-field enhancement at the pinholes is almost the same as that for the pinhole-free nanoshells. Variation of hole sizes from 2 to $5 \mathrm{~nm}$ leads to similar E-field enhancement predictions (though the location of maximum enhancement varies). All of the calculations above consider cylindrical shaped holes on the nanoshells; further variation of hole shapes (such as ellipse and triangular prism as shown in supporting materials, Figure S3) also leads to similar E-fields enhancement predictions.

For Ag rods, Ag spheroids, and Ag triangular prisms, the peak E-fields are calculated (using the same DDA approach described here) to be about 4500, 4700, and 3500 times the applied field, respectively, ${ }^{25}$ so the pinhole enhancements found here are not unusual; however, they do provide a significant effect relative to the pinhole free results.

\section{Conclusion}

We report the synthesis of Au nanoshells via wet-chemical methods. TEM results indicate that the shell is not continuous, resulting in pinhole (several $\mathrm{nm}$ ) structures. Using the discrete dipole approximation and Mie theory, we find that the optical properties of metal nanoshells depend strongly on their size and shell thickness. This result is consistent with previous studies of metal nanoshells with dielectric cores. Our DDA calculations indicate that the pinholes have a weak effect on extinction spectra, but they provide hot spots for electromagnetic field enhancement such that $|\mathbf{E}|^{2}$ is $3-4$ times larger or even close to 10 times larger for peak fields. This means that molecules in pinholes will show SERS enhancements that are about 10100 times that for molecules on perfect shells (the square of the E-field enhancement). The E-field enhancement from metal nanoshells is comparable to that of nanoscale triangular prisms, spheroids, and rods. Of course the significance of this result would depend on the pinhole density; however, if more than $10 \%$ of the surface area were pinholes, then the contributions 

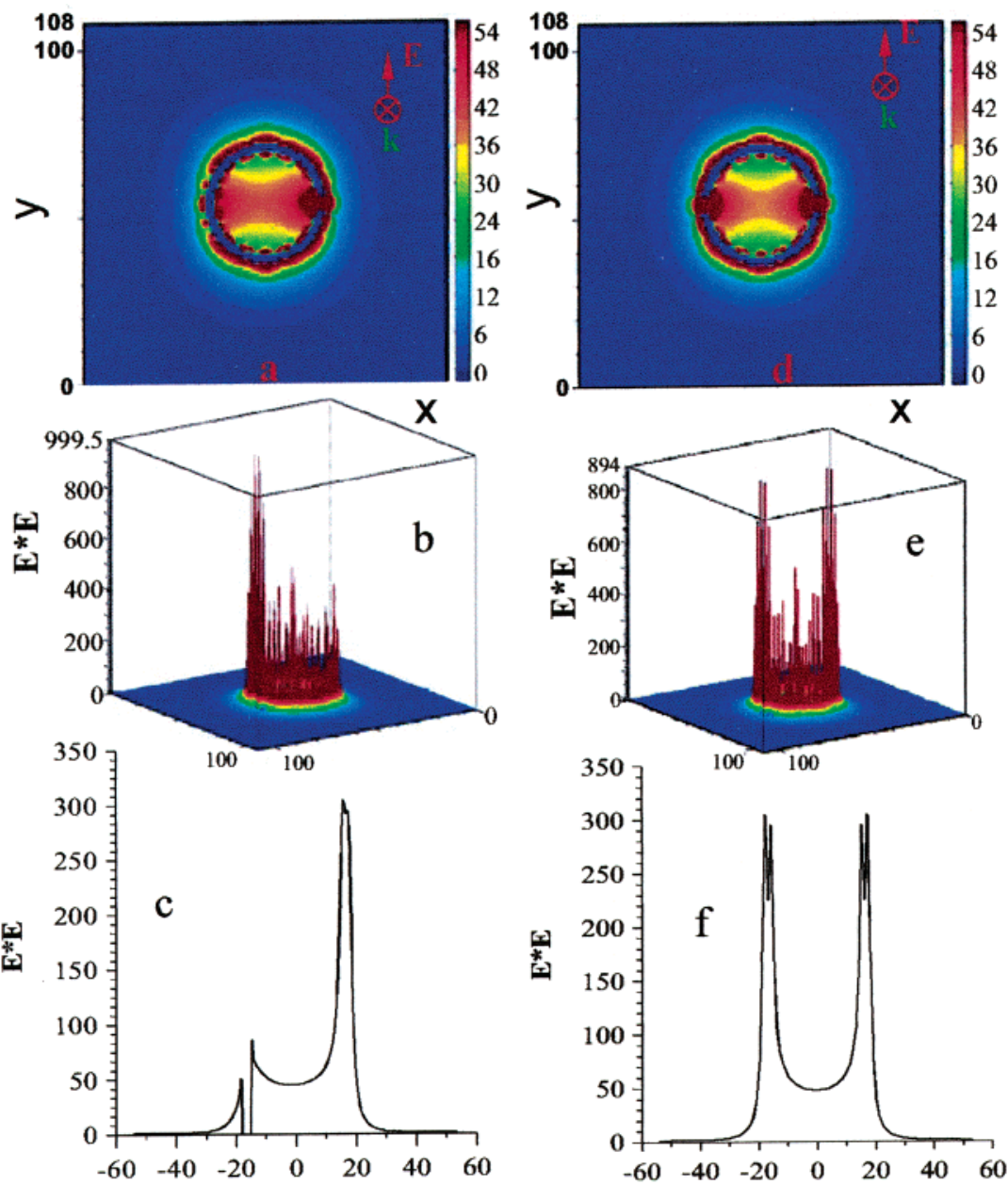

Figure 5. (a) E-field enhancement contours external to a Au nanoshell ( $18 \mathrm{~nm}$ in radii and $3 \mathrm{~nm}$ in thickness) with one hole and wavelength taken to be the plasmon peak, (b) 3-D plot of (a), (c) Line scan analysis of (a) for $y=0$ and along $x$ axis, (d) E-field enhancement contours external to a Au nanoshell with two holes, (e) 3-D plot of (d), (f) Line scan of (d) for $y=0$ and along the $x$ axis. The DDA calculations use a cubic grid with a grid spacing of $1 \mathrm{~nm}$.

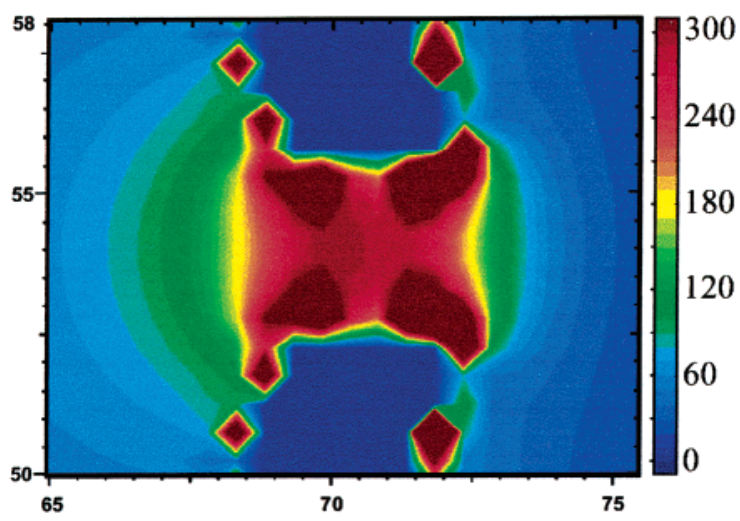

Figure 6. E-field enhancement contours for the hole region associated with Figure 6, parts a and b.

of pinholes to the SERS measurements could exceed that of the rest of the surface.

Acknowledgment. We gratefully acknowledge support from the National Science Foundation through the Materials Research Center (MRSEC). Support was also provided by the Air Force Office of Scientific Research MURI program (F49620-02-10381).
Supporting Information Available: TEM photograph of Au nanoshell (Figure S1). AFM image of Au nanoshells and cross-sectional topographic analysis of the Au nanoshells (Figure S2). E-field enhancement contours external to a Au nanoshell (Figure S3). This material is available free of charge via the Internet at http://pubs.acs.org.

\section{References and Notes}

(1) Yu, Y.-Y.; Chang, S.-S.; Lee, C.-L.; Wang, C. R. C. J. Phys. Chem. B 1997, 101, 6661 617.

(2) Jana, N. R.; Gearheart, L.; Murphy, C. J. Chem. Commun. 2001,

(3) Link, S.; El-Sayed, M. A. J. Phys. Chem. B 1999, 103, 8410

(4) Kim, F.; Song, J. H.; Yang, P. J. Am. Chem. Soc. 2002, 124, 14316.

(5) Jin, R.; Cao, Y.; Mirkin, C. A.; Kelly, K. L.; Schatz, G. C.; Zhang, J. G. Science 2001, 294, 1901.

(6) Sun, Y.; Mayers, B.; Xia, Y. Nano Lett. 2003, 3, 675.

(7) Hao, E.; Kelly, K. L.; Hupp, J. T.; Schatz, G. C. J. Am. Chem. Soc. 2002, 124, 15182

(8) Chen, S.; Fan, Z.; Carroll, D. L. J. Phys. Chem. B 2002, 106, 10777.

(9) Oldenburg, S. J.; Averitt, R. D.; Westcott, S. L.; Halas, N. J. Chem. Phys. Lett. 1998, 288, 243.

(10) Oldenburg, S. J.; Jackson, J. B.; Westcott, S. L.; Halas, N. J. Appl. Phys. Lett. 1999, 75, 2897.

(11) Graf, C.; Blaaderen, A. V. Langmuir 2002, 18, 524

(12) Kelly, K. L.; Coronado, E.; Zhao, L. L.; Schatz, G. C. J. Phys Chem. B 2003, 107, 668; Program DDSCAT, by B. T. Draine and P. J. Flateau. University of California, San Diego, Scripps Institute of Oceanography, 8605 La Jolla Dr., La Jolla, CA 92093-0221.

(13) Yang, W. H.; Schatz, G. C.; Duyne, R. P. V. J. Phys. Chem. 1995 $99,869$. 
(14) Kelly, K. L.; Lazarides, A. A.; Schatz, G. C. Comput. Sci. Eng. 2001, 3, 67.

(15) Jensen, T.; Kelly, K. L.; Lazarides, A.; Schatz, G. C. J. Cluster Sci. 1999, 10, 295.

(16) Bian, R. X.; Dunn, R. C.; Xie, X. S.; Leung, P. T. Phys. Rev. Lett. 1995, 75, 4772 .

(17) Moreno, E.; Erni, D.; Hafner, C.; Vahldieck, R. J. Opt. Soc. A 2002 $19,101$.

(18) Hirsch, L. R.; Jackson, J. B.; Lee, A.; Halas, N. J.; West, J. L. Anal. Chem. 2003, 75, 2377.

(19) Jackson, J. B.; Westcott, S. L.; Hirsch, L. R.; West, J. L.; Halas, N. J. Appl. Phys. Lett. 2003, 82, 257.

(20) Pordan, E.; Nordlander, P. Nano Lett. 2003, 3, 543.

(21) Charnay, C.; Lee, A.; Man, S.-Q.; Moran, C. E.; Radloff, C.; Bradley, R. K.; Halas, N. J. J. Phys. Chem. B 2003, 107, 7327.

(22) Sun, Y.; Xia, Y. Anal. Chem. 2002, 74, 5297

(23) Clays, K.; Hemdrickx, E.; Triest, M.; Persoons, A. J. Mol. Liq. 1995, 67, 133

(24) Palik, E. D. Handbook of Optical Constants of Solids; Academic Press: New York, 1985.

(25) Hao, E.; Schatz, G. C. J. Chem. Phys. 2004, in press.

(26) Xia, Y. N.; Yang, P. D.; Sun, Y. G.; Wu, Y. Y.; Mayers, B.; Gates, B.; Yin, Y. D.; Kim, F.; Yan, Y. Q. Adv. Mater. 2003, 15, 353.

(27) Sun, Y. G.; Xia, Y. N. Science 2002, 298, 2176.
(28) Sun, Y. A.; Xia, Y. N. Adv. Mater. 2003, 15, 695.

(29) Metraux, G. S.; Cao, Y. C.; Jin, R. C.; Mirkin, C. A. Nano Lett. 2003, 3, 519 .

(30) Williams, D. B.; Carter, C. B. Transmission Electron Microscopy IV Spectroscopy; Plenum Press: New York, 1996.

(31) Shaiu, W.-L.; Vesenka, J.; Jondle, D.; Henderson, E.; Larson, D.

P. J. Vac. Sci. Technol. 1993, 11, 820.

(32) Jackson, J. B.; Halas, N. J. J. Phys. Chem. B 2001, 105, 2743.

(33) Mie, G. Ann. Phys. 1908, 25, 377.

(34) Kim, Y.; Johnson, R. C.; Li, J.; Hupp, J. T.; Schatz, G. C. Chem. Phys. Lett. 2002, 352, 421.

(35) Coronado, E.; Schatz, G. C. J. Chem. Phys. 2003, 119, 3926

(36) Nie, S.; Emory, S. R. Science 1997, 275, 1102.

(37) Michaels, A. M.; Nirmal, M.; Brus, L. E. J. Am. Chem. Soc. 1999, 121,9932

(38) Doering, W. E.; Nie, S. J. Phys. Chem. B 2002, 106, 311.

(39) Schatz, G. C. Acc. Chem. Res. 1984, 17, 370.

(40) Inoue, M.; Ohtaka, K. J. Phys. Soc. JPN 1983, 52, 3853

(41) Xu, H.; Bjerneld, E. J.; Käll, M.; Borjesson, L. Phys. Rev. Lett. 1999, 83, 4357.

(42) Xu, H.; Aizpurua, J.; Käll, M.; Apell, P. Phys. Rev. E 2000, 62, 4318.

(43) Michaels, A. M.; J, J.; Brus, L. E. J. Phys. Chem. B 2000, 104, 11965 